\title{
A Deeply Virtual Compton Scattering Amplitude
}

\section{Capua:"T}

Dipartimento di Fisica, Università della Calabria and INFN - Gruppo Collegato di Cosenza

I-87036 Rende, Italy

E-mail: capua@Cs.infn.it

A factorized Regge-pole model for deeply virtual Compton scattering is suggested. Fits to the existing HERA data on deeply virtual Compton scattering are presented.

International Workshop on Diffraction in High-Energy Physics-Diffraction 06-

Adamantas, Milos island, Greece

5-10 September 2006

Speaker

* In collaboration with S. Fazio, R. Fiore, L.L.Jenkovszky, F. Paccanoni 


\section{Introduction}

Interest in deeply virtual Compton scattering (DVCS) is related to the prospects to use it as a tool in studies of Generalized Parton Distributions (GPD) [1,2].

At HERA the DVCS cross-section has been measured $[3,4]$ in diffractive ep interactions, as a function of $Q^{2}, W$ and $t$ that are respectively the photon virtuality, the invariant mass of the $\gamma^{*} p$ system and the squared 4-momentum trasfered at the proton vertex. Since the electronproton scattering at HERA is dominated by a single photon exchange, the calculation of the DVCS scattering amplitude reduces to that of the $\gamma^{*} p \rightarrow \gamma p$ amplitude, which at high energies, in the Regge-pole approach, is dominated by the exchange of positive-signature Reggeons. The diagrams in Fig. 1 show the production of a real photon at HERA (left) and the DVCS amplitude in a Regge-factorized form (right). In the figure, $q_{1,2}$ are the four-momenta of the incoming and outgoing photons, $p_{1,2}$ are the four-momenta of the incoming and outgoing protons, $r$ is the fourmomentum of the Reggeon exchanged in the $t$ channel, $r^{2}=t=\left(q_{1}-q_{2}\right)^{2}$ and $s=W^{2}=\left(q_{1}+p_{1}\right)^{2}$ is the squared centre-of-mass energy of the incoming system.
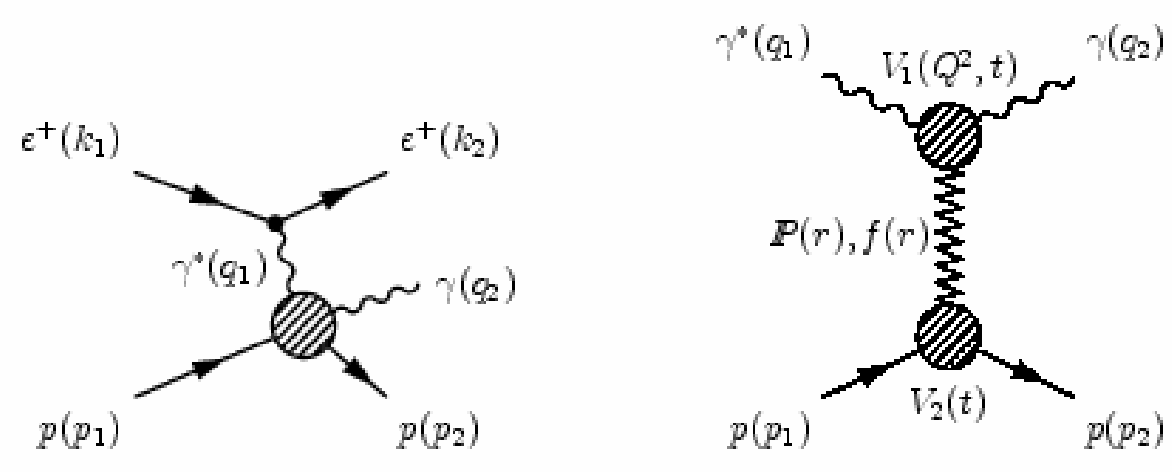

Figure 1: Diagram of a DVCS process at HERA (left); DVCS amplitude in a Reggefactorized form (right).

This contribution shows the comparison of the HERA DVCS data with an explicit model for DVCS with $Q^{2}$ - and $t$-dependences determined by the $\gamma^{*} p \gamma$ vertex. A detailed description of the model, briefly presented in the next section, can be found in [5].

\section{The model}

Unless specified (as in the deep inelastic scattering (DIS) limit), $q^{2}=0$, and hence, for brevity, $q_{1}^{2}=-Q^{2}$. In the upper vertex $V_{1}$, see Fig. 1 , a virtual photon with 4-momentum $q_{1}$, and a Reggeon (e.g. Pomeron) with 4-momentum $r$, enters and a real photon, with 4-momentum $q_{2}=q_{1}+r$ appears in the final state as an outgoing particle. The vertex $V_{l}$ depends on all the possible invariants constructed with the above 4-momenta, $V_{1}\left[q_{1}^{2}, r^{2}, q_{1} \cdot r\right]$, where $r^{2}=t<0$, $q_{1}^{2}=Q^{2}<0$. The three invariants are not independent since the mass-shell condition for the outgoing photon, $q_{2}^{2}=\left(q_{1}+r\right)^{2}=0$, provides the relation $q_{1} \cdot r=-q_{1}^{2}-r^{2} / 2=Q^{2}-t / 2$. 
Hence, the vertex can be considered as a function of the invariants $\left[Q^{2}, q_{1} \cdot r\right]$ or $\left[t, q_{1} \cdot r\right]$. This does not mean that the variables cannot appear separately, but it could also happen that $q_{1} \cdot r$ becomes a scaling variable, and consequently the vertex will finally depend on $q_{l} \cdot r$ only. It depends on the dynamics of the process and, for the moment, we prefer to keep $t$, apart from $Q^{2}$, as the second independent variable.

The interplay of the $Q^{2}$ - and $t$-dependence in the DVCS amplitude was recently discussed in Ref.[6], where the existence of a new, universal variable $z$ was suggested. The basic idea is that $Q^{2}$ and $t$, both having the meaning of a squared mass of a virtual particle (photon or Reggeon), should be treated on the same footing, by means a new variable, defined as

$$
z=q_{1}^{2}+t=-Q^{2}+t
$$

This variable can be generalized to vector meson electroproduction $z=t-\left(Q^{2}+M_{V}^{2}\right)$ or virtual photon (lepton pair) electroproduction, $z=t-\left(Q_{1}{ }^{2}+Q_{2}{ }^{2}\right)$, where $Q_{2}{ }^{2}=-q_{2}{ }^{2}$. However, differently from Ref. [6], here we introduce the new variable only in the upper, $\gamma * I P \gamma$ vertex, to which the photons couple. According to the Fig.1, the DVCS amplitude can be written as

$$
A\left(s, t, Q^{2}\right)_{\gamma^{*} p \rightarrow p p}=-A_{0} V_{l}\left(t, Q^{2}\right) V^{2}(t)\left(-i s / s_{0}\right)^{\alpha(t)},
$$

where $A_{0}$ is a normalization factor, $V_{I}\left(t, Q^{2}\right)$ is the $\gamma^{*} I P \gamma$ vertex, $V_{2}(t)$ is the $p I P p$ vertex and $\alpha(t)$ is the exchanged Pomeron trajectory, which we assume in a logarithmic form:

$$
\alpha(t)=\alpha(0)-\alpha_{1} \ln \left(1-\alpha_{2} t\right)
$$

Such a trajectory is nearly linear for small $|t|$, thus reproducing the forward cone of the differential cross section, while its logarithmic asymptotics provides for the large-angle scaling behaviour [7,8], typical of hard collisions at small distances, with power-law fall-off in $|t|$, obeying quark counting rules [7, 9,10]. Here we are referring to the dominant Pomeron contribution plus a secondary trajectory, e.g. the $f$-Reggeon. Although we are aware of the importance of this subleading contribution at HERA energies, nevertheless we cannot afford the duplication of the number of free parameters, therefore we include it effectively by rescaling the parameters. Ultimately, the Pomeron and the $f$-Reggeon have the same functional form, differing only by the values of their parameters. Furthermore, the Pomeron [11] itself is unlikely to be a single term, so instead of including several Regge terms with many free parameters, it may be reasonable to comprise them in a single term, called effective Reggeon or effective Pomeron, depending on the kinematical region of interest. Although the parameters of this effective Reggeon (Pomeron) (e.g. its intercept and slope) can be close to the one (whose form is at best a convention), for the above reason they never should be taken as granted.

For convenience, and following the arguments based on duality (see Ref. [12] and references therein), the $t$ dependence of the $p I P p$ vertex is introduced via the $\alpha(t)$ trajectory: $V_{2}(t)=e^{b \alpha(t)}$, where $b$ is a parameter.

A generalization of this concept will be applied also to the upper, $\gamma^{*} I P \gamma$ vertex by introducing the trajectory $\beta(z)=\alpha(0)-\alpha_{1} \ln \left(1-\alpha_{2} z\right)$, where the value of the parameter $\alpha_{2}$ may 
be different in $\alpha(t)$ and $\beta(z)$ (a relevant check will be possible when more data will be available). Hence the scattering amplitude (6), with the correct signature, becomes

$$
A\left(s, t, Q^{2}\right)_{\gamma^{*} p \rightarrow \gamma p}=-A_{0} e^{b \alpha(t)} e^{b \beta(z)}\left(-i s / s_{0}\right)^{\alpha(t)}=-A_{0} e^{(b+L)} \alpha(t)+b \beta(z),
$$

where $L \sim \ln \left(-i s / s_{0}\right)$.

The model contains a limited number of free parameters. Moreover, most of them can be estimated a priori. The product $\alpha_{1} \alpha_{2}$ is just the forward slope $\alpha^{\prime}$ of the Reggeon $\left(\sim 0.2 \mathrm{GeV}^{-2}\right.$ for the Pomeron, but much higher for $f$ and/or for an effective Reggeon). The value of $\alpha_{1}$ can be estimated from the large-angle quark counting rules [7,9,10]. For large $t\left(|t|>>1 \mathrm{GeV}^{2}\right)$ the amplitude goes roughly (a detailed treatment can be found in Refs. $[7,8])$ like $\sim \exp \left[-\alpha_{1} \ln (-t)\right]=($ $-t)^{-\alpha l}$, where the power $\alpha_{l}$ is related to the number of quarks in a collision [7,9,10], e.g. their number minus one. We set $\alpha_{1}=1$, and hence $\alpha_{2}=\alpha$ '. For the intercept of our effective Reggeon, dominated by the Pomeron, we set $\alpha(0)=1.25$ as an average over the soft+hard Pomerons.

The above values of the parameters should not be taken as granted, they should be considered as starting values in the fitting procedure. From Eq. (1) the slope of the forward cone is

$$
B\left(s, Q^{2}, t\right)=d / d t \ln |A|^{2}=2\left[b+\ln \left(s / s_{0}\right)\right] \alpha^{\prime} /\left(1-\alpha_{2} t\right)+2 b \alpha^{\prime} /\left(1-\alpha_{2} z\right),
$$

which, in the forward limit, $t=0$ reduces to

$$
B\left(s, Q^{2}\right)=2\left[b+\ln \left(s / s_{0}\right)\right] \alpha^{\prime}+2 b \alpha^{\prime} /\left(1+\alpha_{2} Q^{2}\right) .
$$

Thus, the slope shows shrinkage in $s$ and antishrinkage in $Q^{2}$. Details of this model can be found in [5].

\section{Fits and results}

A standard procedure for the fit to the HERA data on DVCS [3,4] based on Eq. (1) has been adopted. A detailed analysis of the data would require a sum of a Pomeron plus an $f$ Reggeon contribution: $A=A^{P}+A^{f}$.

To avoid the introduction of too many parameters, given the limited number of experimental data points, we use a single Reggeon term, which can be treated as an effective Reggeon. The parameters $\alpha(0), \alpha_{1}$ and $\alpha^{\prime}$ have been fixed to $1.25,1.0$ and $0.38 \sim \mathrm{GeV}^{-2}$ respectively and the values of the fitted parameters $A_{0}$ and $b$, described in Eq. (1) are listed in Table 1 . The value of $\alpha^{\prime}$ has been determined in an exploratory fit with this parameter left free to vary between 0.2 and $0.4 \sim \mathrm{GeV}^{-2}$.

The ZEUS measurements have been rescaled to the $W$ and $Q^{2}$ values of the H1 measurements. The mean value of $|t|$ has been fixed to $0.17 \mathrm{GeV}^{2}$ according with the $\mathrm{H} 1$ measurements of the differential cross-section in the range $(0.1-0.8) \mathrm{GeV}^{2}$ for $\mathrm{H} 1$ [3] taking into account the value $6.02 \mathrm{GeV}^{-2}$ for the slope $\mathrm{B}$ as determined by the experiment. 



Figure 2: The $\gamma^{*} p \rightarrow \gamma p$ cross section as a function of $Q^{2}(a)$, of $W(b)$ and the cross section differential in $t$ (c) measured by HI and ZEUS [3,4]. The ZEUS measurements have been rescaled to the $\mathrm{H} 1$ values. The lines show the results of the fits obtained from $E q(4)$ to the data.

\begin{tabular}{|c|c|c|c|}
\hline Parameter & $\boldsymbol{\sigma}_{\mathrm{DvCS}} \mathbf{v s} \mathbf{Q}^{2}$ & $\boldsymbol{\sigma}_{\mathrm{DVCS}} \mathbf{v s} \mathbf{t}$ & $\boldsymbol{\sigma}_{\mathrm{DVCS}} \mathbf{v s} \mathbf{W}$ \\
\hline$\left|\boldsymbol{A}_{\boldsymbol{0}}\right|^{2}$ & $0.08 \pm 0.01$ & $0.11 \pm 0.24$ & $0.06 \pm 0.01$ \\
\hline $\boldsymbol{B}$ & $0.93 \pm 0.05$ & $1.04 \pm 0.91$ & $1.08 \pm 0.10$ \\
\hline$\chi^{2} /$ ndof & 0.57 & 0.15 & 1.15 \\
\hline
\end{tabular}

Table 1: The values of the fitted parameters quoted in Eq. (1).

The results of the fits to the HERA data on DVCS are shown in Fig. 2. The cross-section $\sigma\left(\gamma^{*} p \rightarrow \gamma p\right)$ as a function of $Q^{2}$ and $W=\sqrt{s}$ are presented respectively in Fig. 2a and Fig. 2b. The differential cross-section $d \sigma\left(\gamma^{*} p \rightarrow \gamma p\right) / d t$, given by

$$
d \sigma / d t\left(s, t, Q^{2}\right)=\pi / s^{2}\left|A\left(s, t, Q^{2}\right)\right|^{2},
$$


is presented in Fig. 2c. The quality of the fits is satisfactory; in particular our model fits rather well the cross-sections as a function of $Q^{2}$ and the cross-section differential in $t$. Although the present HERA data on DVCS are well within the "soft" region, the model potentially is applicable for much higher values of $|t|$, dominate by hard scattering. Finally, Fig. 3 shows antishrinkage in $Q^{2}$ and shrinkage in $W$ of the forward cone, according to Eqs. 2 and 3. The curves are compared with the $\mathrm{H} 1$ experimental results.
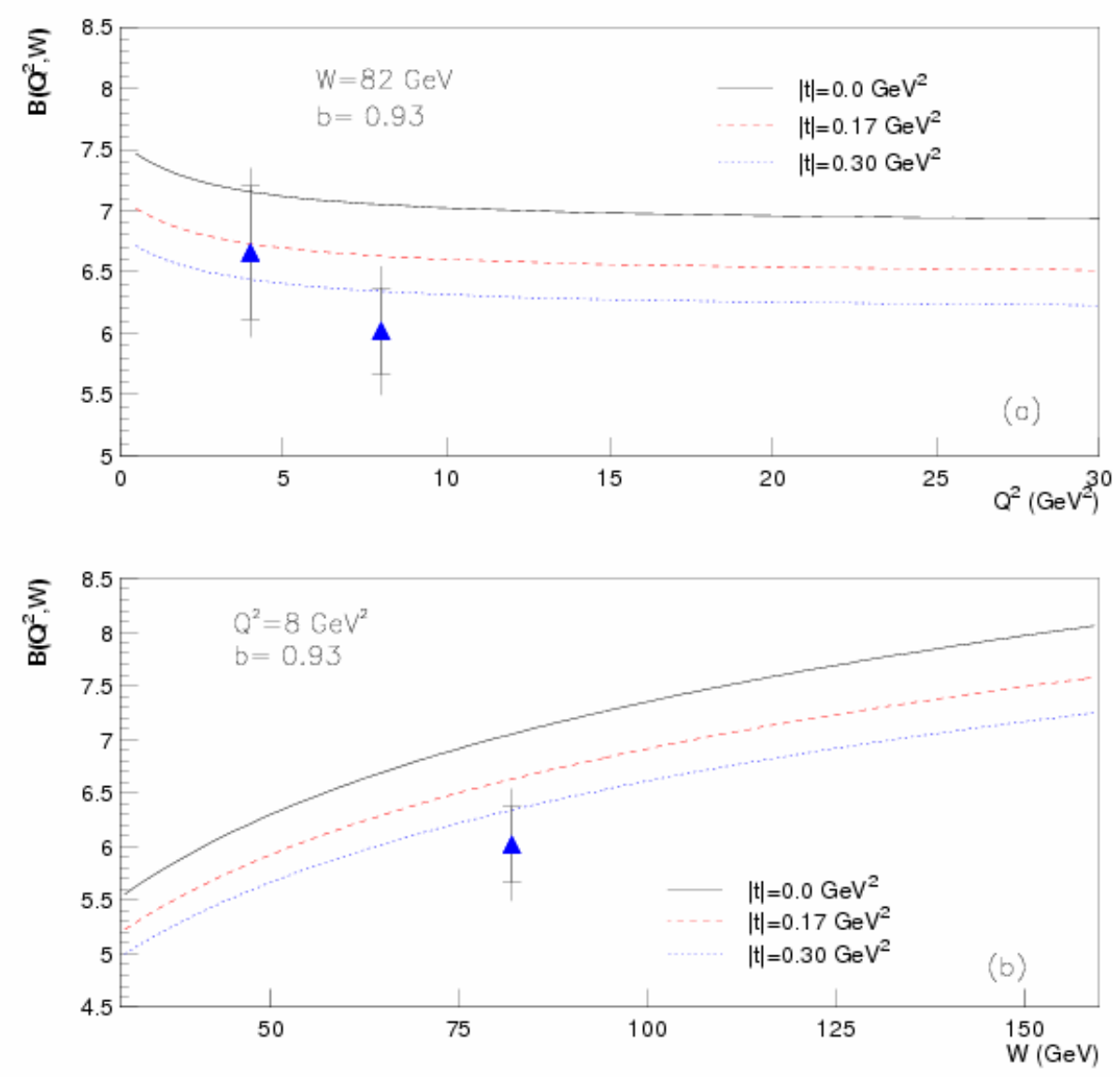

Figure 3: The $Q^{2}$ - and s dependence of the local slope described in Eq. (2) (dotted and dashed line) and Eq. (3) (solid line). The triangles show the experimental measurements of $H 1$.

\section{Conclusions}

A fit based on the [5] model has been compared with the HERA data on DVCS. The quality of the fits is satisfactory.

The fits to the data could be improved, when more data are available, by accounting for the Pomeron(s) and $f$-Reggeon contributions separately as well as by using expressions for Regge trajectories which take exactly into account analyticity and unitarity. On the other hand, the model can be used to study various extreme regimes of the scattering amplitude in all the three variables it depends on. 
The transition from Regge behaviour to QCD evolution at large $Q^{2}$ should be accounted for. A formula interpolating between the two regimes (Regge pole and asymptotic QCD evolution) was proposed in Ref.[13] for $t=0$ only. Its generalization to non zero $t$ value is possible by applying the ideas and the model presented in this paper. The applicability of the model in both soft and hard domains can be used to learn about the transition between perturbative (QCD) and non-perturbative (Regge poles) dynamics.

\section{References}

[1] F.M. Dittes, D. Mueller, D. Robaschik, B. Geyer and J. Horejsi, Phys.Lett. B 209 (1998) 325; D. Mueller, D. Robaschik, B. Geyer, F. M. Dittes and J. Horejsi, Fortsch. Phys. 42 (1994) 101.

[2] X. Ji, Phys. Rev. Lett. 78 (1997) 610; A.V. Radyushkin, Phys. Lett. B 380 (1996) 417; M. Diehl, Phys. Rept. 338 (2003) 41.

[3] H1 Coll.: A. Aktas et al., Eur. Phys. J. C 44 (2005) 1.

[4] ZEUS Coll.: S. Chekanov et al., Phys. Lett. B 573 (2003) 46.

[5] L.L. Jenkovszky, these proceedings; M. Capua, S. Fazio, R. Fiore, L.L. Jenkovszky and F. Paccanoni; Phys. Lett. B 645 (2006) 161.

[6] R. Fiore, L. Jenkovszky, V. Magas, and A. Prokudin, Interplay between $Q^{2}$ - and $t$ - dependences in deeply virtual Compton scattering, In the Proceedings of the Crimean conference New Trends in High-Energy Physics, Yalta, 10-17 September 2005.

[7] D.D. Coon, J.F. Gunion, Tran Thanh Van, R. Blankenbecler, Phenomenology of High Momentum Transfer Elastic Processes, SLAC-PUB-1483 (September 1974), Phys. Rev. D 18 (1978) 1451.

[8] L.L. Jenkovszky, Z.E. Chikovani, Yad. Fizika 30 (1979) 531 (English translation: Sov. J. Nucl. Phys.); A.I. Bugrij, Z.E. Chikovani, L.L. Jenkovszky, Z. Phys. C, Particles and Fields, 4 (1980) 45; L.L. Jenkovszky, Nucl. Phys. B (Proc. Suppl.) 12 (1990) 317; R. Fiore, L.L. Jenkovszky, V.K. Magas, and F. Paccanoni, Phys. Rev. D 60 (1999) 116003.

[9] V.A. Matveev, R.M. Muradyan, A.N. Tavkhelidze, Lett. Nuovo Cim, 7 (1973) 719.

[10] S. Brodsky and G. Farrar, Phys. Rev. Lett. 31 (1973) 1153.

[11] V.S. Fadin, E.A. Kuraev, L.N. Lipatov, Phys. Lett. B 60 (1975) 50; I.I. Balitsky, L.N. Lipatov, Yad. Fizika 28 (1978) 822.

[12] L.L. Jenkovszky, Rivista Nuovo Cim., 10 (1987) 1.

[13] P. Desgrolard, L.L. Jenkovszky, E. Paccanoni, Eur. Phys. J. C 7 (1999) 263. 